\title{
Preliminary results of feasibility of self-calibration of silicon $p n$ photodiodes at room temperature using temperature sensors
}

\section{Resultados preliminares de viabilidad para la auto-calibración de fotodiodos pn de silicio a temperatura ambiente empleando sensores de temperatura}

\author{
E. Borreguero ${ }^{1 *}$, C. K. Tang 2 , J. Gran ${ }^{2}$, A. Pons ${ }^{1, \mathrm{~S}}$, J. Campos ${ }^{1, \mathrm{~S}}$. \\ 1. Instituto de Óptica "Daza de Valdés" (IO-CSIC), Serrano 121, 28006 Madrid, Spain \\ 2. Justervesenet (JV), PO Box 170, 2027 Kjeller, Norway \\ ${ }^{(*)}$ E-mail: elisa.borreguero@csic.es \\ S: SEDOPTICA member \\ Received: 17/07/2017_Accepted: 09/05/2018 \\ DOI: $10.7149 /$ OPA.51.2.50013
}

\begin{abstract}
:
Predictable quantum efficient detectors (PQEDs), based on photoelectric effect in silicon semiconductor, have proved their potential as optical radiant power primary standard. At present, simulations for semiconductor devices are the common method to predict the response of a PQED. As an alternative, this work investigates the feasibility of an experimental technique, the self-calibration of silicon photodiodes at room temperature by means of temperature sensors. A device which compares two physics principles by optical power, working both as a photodetector and a radiometer based on electrical substitution, was made. The device allows for measuring the current generated under irradiation (in photocurrent mode) and the heating of the system, induced by a laser or forward biasing, to determine the optical power (in thermal mode). The feasibility study consists of testing the equivalence between both modes and establishing the suitable range of optical power. The range of optimum optical power level, where both modes run in the linear domain at the present configuration, is from $300 \mu \mathrm{W}$ up to $1 \mathrm{~mW}$. Within this range, the optical power experimental uncertainty is lower than $1 \%$.
\end{abstract}

Key words: self-calibration, radiometer, silicon photodiode, PQED.

\section{RESUMEN:}

Los detectores cuánticos, eficientes y predecibles (PQEDs), basados en el efecto fotoeléctrico del silicio como material semiconductor, han demostrado su potencial como patrón primario de flujo radiante. Hasta ahora, las simulaciones con programas de dispositivos semiconductores son el método común para predecir la respuesta de un PQED. Como alternativa, este trabajo investiga la viabilidad de una técnica experimental, la auto-calibración de fotodiodos de silicio a temperatura ambiente mediante sensores de temperatura. Se construyó un dispositivo que compara dos principios físicos a través de la potencia óptica, funcionando como fotodetector y como radiómetro basado en la sustitución eléctrica. El dispositivo permite medir la corriente generada por la irradiancia cuando es iluminado (en modo fotocorriente) y el calentamiento del sistema, inducido mediante un láser o por polarización eléctrica directa, para determinar la potencia óptica (en modo térmico). El estudio de viabilidad consiste en comprobar la equivalencia entre los dos modos y establecer el rango apropiado de potencia óptica para ello. El rango de nivel óptimo de potencia óptica, donde ambos modos operan en el dominio lineal con la configuración actual, comprende desde $300 \mu \mathrm{W}$ hasta $1 \mathrm{~mW}$. Dentro de este rango, la incertidumbre experimental de la potencia óptica es menor que el $1 \%$.

Palabras clave: auto-calibración, radiómetro, fotodiodo de silicio, PQED. 


\section{REFERENCES AND LINKS / REFERENCIAS Y ENLACES}

[1] EMRP European project, "Candela: Towards quantum based photon standards," http://www.quantumcandela.org

[2] EMRP European project, "New primary standard and traceability for radiometry," http://www.inrim.it/Newstar/project.org

[3] M. Sildoja, F. Manoocheri, M. Merimaa, E. Ikonen, I. Müller, L. Werner, J. Gran, T. Kübarsepp, M. Smîd, M.L. Rastello, "Predictable quantum efficient detector: I. Photodiodes and predicted responsivity," Metrologia 50, 385-394 (2013).

[4] I. Müller, U. Johannsen, U. Linke, L. Socaciu-Siebert, M. Smîd, G. Porrovecchio, M. Sildoja, F. Manoocheri, E. Ikonen, J. Gran, T. Kübarsepp, G. Brida, L. Werner, "Predictable quantum efficient detector: II. Characterization and confirmed responsivity," Metrologia 50, 395-401 (2013).

[5] T. E. Hansen, "Silicon UV-photodiodes using natural inversion layers," Physica Scripta 18, 471-475 (1978).

[6] P. V. Foukal, C.Hoyt, H. Kochling, P. Miller, "Cryogenic absolute radiometers as laboratory irradiance standards, remote sensing detectors, and pyroheliometers," Applied Optics 29, 988-993 (1990).

[7] J. Gran, T. Kübarsepp, M. Sildoja, F. Manoocheri, E. Ikonen, I. Müller, "Simulations of a predictable quantum efficient detector with PC1D," Metrologia 49, 130-134 (2012).

[8] M. White, J. Gran, N. Tomlin, J. Lehman, "A detector combining quantum and thermal primary radiometric standards in the same artefact," Metrologia 51, 245-251 (2014).

[9] M. U. Nordsveen, C. K. Tang, J. Gran, "Demonstration of a dual-mode Si detector as a self-calibrating device at room temperature," Optics Express 25, 8459-8469 (2017).

[10] J. Campos, J. L. Fontecha, A. Pons, A. Hanson, D. Williams, J. Verrill, "NPL-CSIC comparison of regular reflectance measurements," Metrologia 37, 323-327 (2000).

\section{Introduction}

The response of a photodiode can be characterized through its internal quantum efficiency (IQE). The IQE represents the number of electrons that contribute to the measured photocurrent versus the number of photons absorbed; hence, the ideal value of IQE is one and it would mean absence of internal losses. Then, the spectral responsivity, the electrical response per unit of incident optical power, can be determined by:

$$
R(\quad)=\frac{I_{p h}}{P_{o p t}}=\frac{e n}{h c} \quad\left[\begin{array}{ll}
1 & (\quad)
\end{array}\right],
$$

where $I_{p h}$ is the generated photocurrent, $P_{o p t}$ the incident optical power, $e$ the elementary charge constant, $n$ the index of refraction of air, $\lambda$ the wavelength of the incident radiation in air, $h$ is Planck's constant, $c$ the speed of light in vacuum, $\eta_{i}$ the internal quantum efficiency of the photodiode and $\rho$ the spectral reflectance of this.

In order to characterize the response of a photodiode, the reflectance can be measured or estimated by models. It is possible to design devices where negligible reflectance losses can be assumed. Furthermore, the IQE can be estimated by models based on hypothesis.

In the last years, within the European joint projects 'Qu-Candela' [1] and 'Newstar' [2], a predictable quantum efficient detector (PQED) [3, 4] was developed and tested. The manufacture specifications of the detectors fulfil the design hypothesis and, therefore, their IQE can be predicted by means of models. As a trap-detector based on induced-junction photodiodes [5], the PQED has demonstrated its potential of being a primary standard of optical radiant power through comparison to the cryogenic electrical substitution radiometer (ESR) [6]. The difference between the two devices is within the uncertainty of the ESR and, hence, other methods are required to validate the estimation of the IQE of a PQED.

At present, software simulations for semiconductor devices are the common method to predict the response of a PQED [7]. No simple experimental techniques exist for an independent validation of a PQED, but a demonstration of a new principle at cryogenic temperatures is conducted [8] and some measurements for 'Qu-Candela' induced-junction photodiodes have been performed at room temperature [9]. The idea is to 
heat the photodiode by forward biasing in order to provide the electrical substitution power equivalent to the optical power.

Based on this idea, this work presents a test device in which, instead of using electrical substitution to calibrate the optical power inducing the same temperature change by electrical and optical power, is more practical to employ three slightly different levels of power to heat a pn photodiode. Two of these levels of power are originated from forward biasing and, taking them as a reference, the other one from an incoming laser. Thus, the optical power is linked to fundamental constants through two different modes within one device and the IQE of the photodiode can be calculated from (1) enabling the self-calibration of the photodiode.

In this background, this work presents a feasibility study to predict the IQE of photodiodes with an experimental technique, at room temperature, based on temperature sensors.

\section{The device}

This device allows for running the photodiode in two different modes of operation: in photocurrent mode and thermal mode, allowing to measure the short-circuit photocurrent generated under irradiation and the electrical heating equivalent to that produced by the absorbed radiation keeping the photodiode in open circuit.

The device consists of three systems: thermal, optical and electrical systems.

\section{2.a. Thermal, electrical and optical components.}

In the optical system, a bare chip $p n$ photodiode and an aluminium mirror placed in an angle of $15^{\circ}$ relative to the photodiode provide multiple reflections of the incident beam to impinge the photodiode, minimizing reflection losses of the optical power. The Fig. 1 shows the optical path followed by the radiation inside the device assembled.

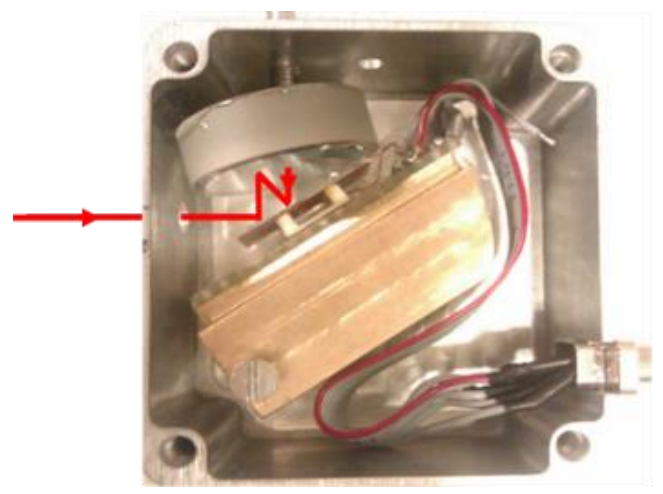

Fig. 1. Picture of the device without electrical connections, indicating the optical path followed by radiation inside the device.

For the thermal system, shown in Fig. 2, two negative temperature coefficient (NTC) thermistors were chosen as temperature sensors because of their variable resistance with temperature. NTC thermistors made of semiconductors are the most sensitive and they are the suitable sensors to the aim of measuring the temperature change in the photodiode as an electrical signal. Catheter germanium NTC thermistors were selected because of its high sensitivity $\left(-5.3 \% /{ }^{\circ} \mathrm{C}\right)$, low room temperature resistance $(1.1 \mathrm{k} \Omega)$ and a small size.

A commercial varnish was employed to thermally anchor the thermistors: one is directly glued to the back side of the photodiode to monitor its temperature, while the second one is attached to a brass socket fastened to the container of the device, which works as heat sink. Thus, the difference between the two thermistors measures the temperature change in the photodiode induced by optical or electrical heating.

A printed circuit board (PCB) was mounted in the brass socket, not only to make the wiring connections but to work both as a photodiode holder and a heat-link. To make this link weak, a small PLA plastic piece is introduced between the photodiode and the PCB. The weaker the heat-link is, the higher the sensitivity and 
the time constant will be. Therefore, it is necessary to find a trade-off value in order to achieve an acceptable signal-to-noise ratio, within a reasonable response time.

In the electrical system, a Wheatstone bridge translates the induced difference of temperature between the thermistors into a voltage difference. Besides, a voltage source allows to forward bias the photodiode to heat it by Joule's effect, in thermal mode, and to reverse bias it in photocurrent mode. The electrical power dissipated when the photodiode is forward biased is given the product of the voltage applied and current measured. The ammeter also measures the photocurrent when the photodiode is irradiated.

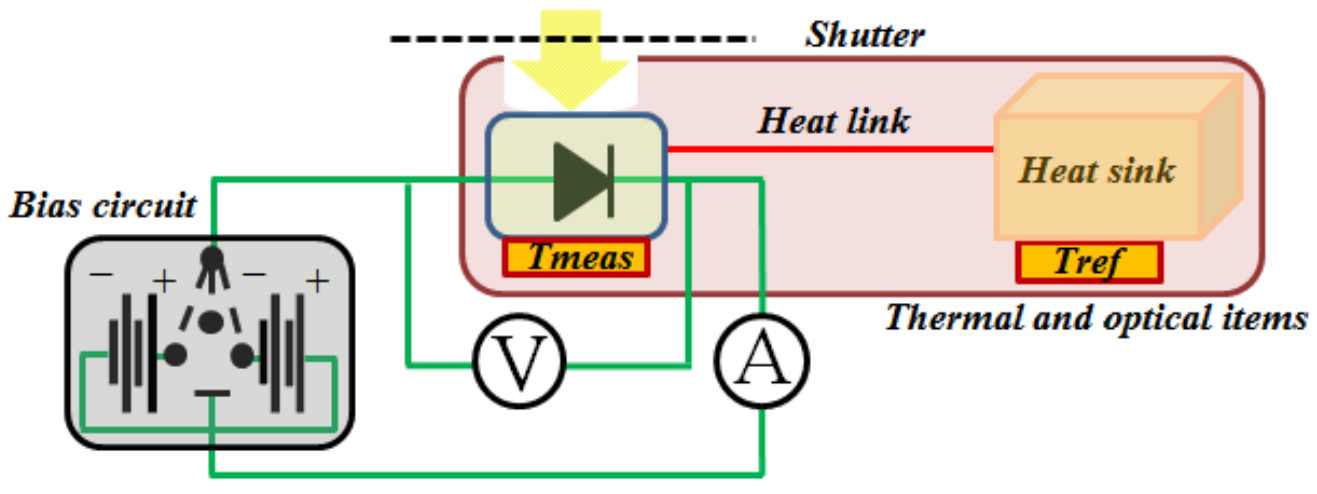

Fig. 2. Device scheme showing thermal, optical and electrical systems. Yellow arrow shows the incoming radiation.

\section{2.b. Characterization of the thermal system response}

The sensitivity and the time constant of the thermal response once the device is assembled, have to be estimated in order to determine the minimum optical power and exposure time that can be measured by the device in thermal mode. On/off cycles forward biasing the device were performed to characterize the response of the system in thermal mode.

A linear combination of two exponentials fit to the output voltage of the Wheatstone bridge corresponding to the average of $90 \mathrm{on} /$ off forward biasing cycles providing $1 \mathrm{~mW}$ heating each, is shown in Fig. 3. It includes the contribution of the power dissipated not only in the photodiode but also in the wiring heat-link and its time constant is around 1 minute.

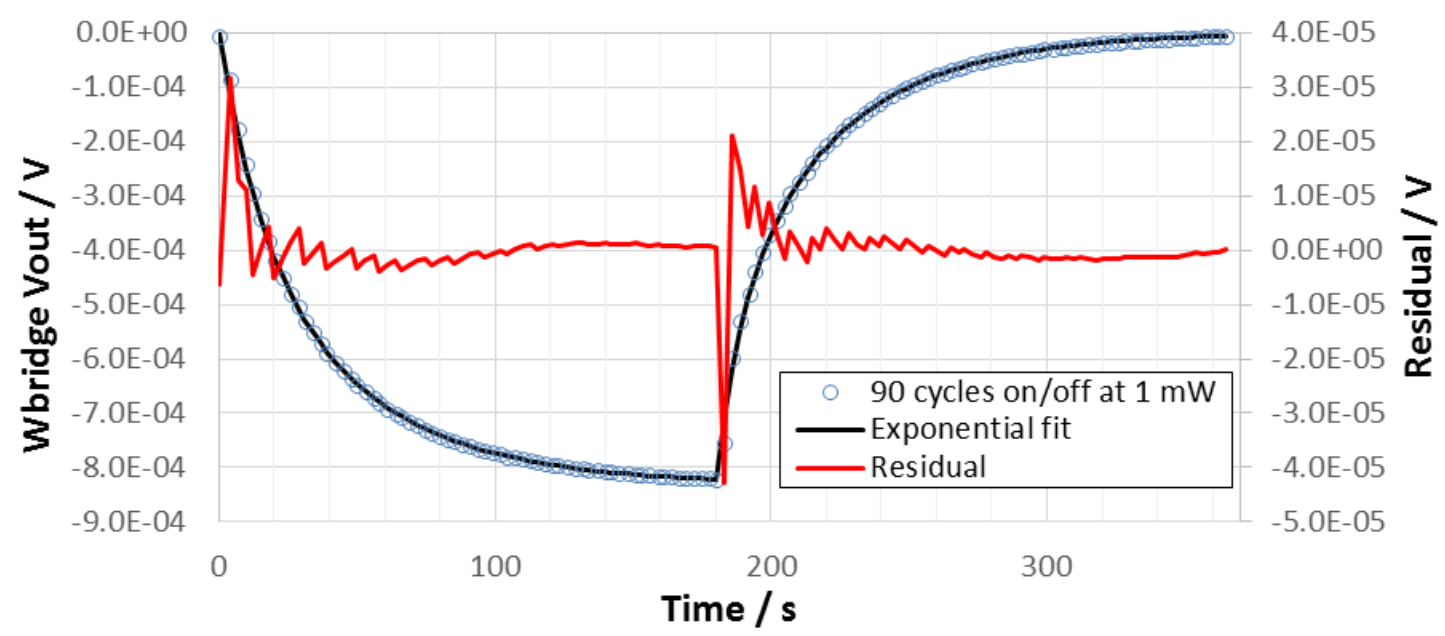

Fig. 3. Exponential fitting of the output voltage of the Wheatstone bridge average of 90 cycles on/off of forward bias voltage that represents the behaviour of the whole system with $1 \mathrm{~mW}$ of electrical power applied. The sensitivity of the device and the signalnoise ratio are in acceptable levels.

The signal amplitude of the Wheatstone bridge versus the dissipated power is plotted in Fig. 4. For the purpose of this work, a linear relation between them can be assumed in the range from $300 \mu \mathrm{W}$ up to $4 \mathrm{~mW}$, as shown in Fig. 4. Lower power values are excluded by the minimum acceptable signal-to-noise ratio and higher values are limited by the nonlinear photocurrent of the photodiode. 


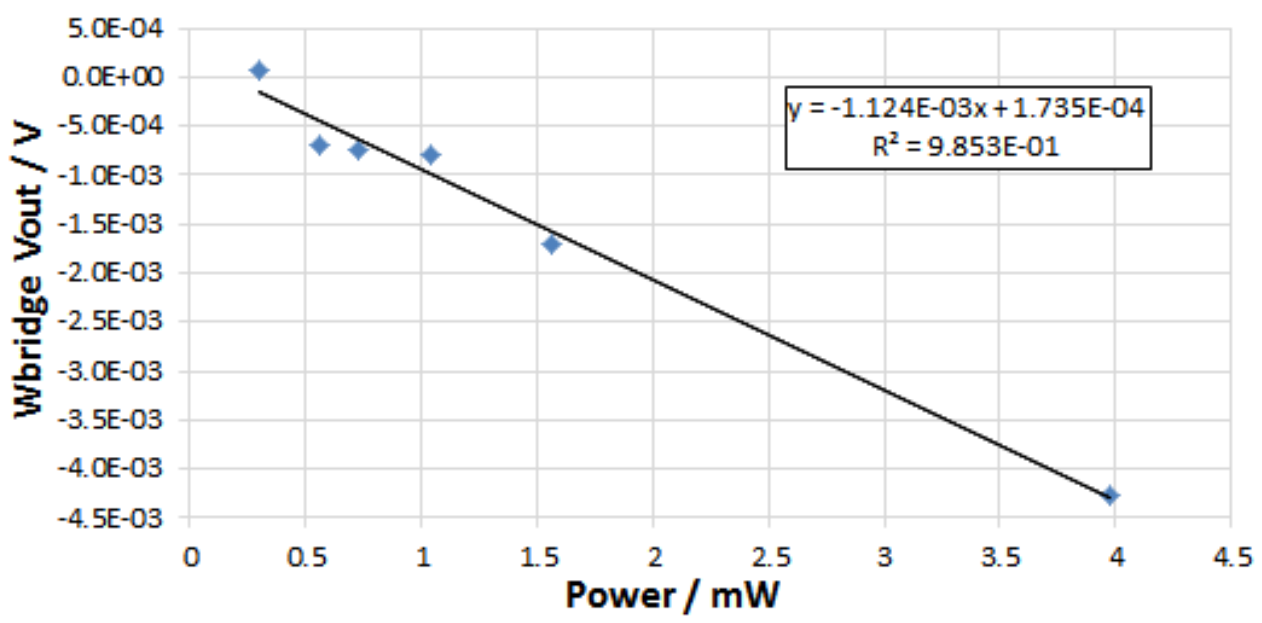

Fig. 4. Linear fitting of the output voltage of the Wheatstone bridge versus forward bias voltage in the range from $300 \mu \mathrm{W}$ to $4 \mathrm{~mW}$.

\section{Self-calibration basis}

In photocurrent mode, where the photons generate electron-hole pairs, the short-circuit current response under irradiation is proportional to the optical power (see equation (1)). Whereas, keeping the photodiode in open circuit under irradiation, the optical power will only heat the device, increasing its temperature and producing an output on the Wheatstone bridge. This heating can be equalized by forward biasing the photodiode in absence of radiation and, since the optical and electrical heating are produced in the same absorber, the photodiode, the electrical power and the optical power can be considered equivalent.

Hence, assuming a linear relationship between the bridge's output voltage and the dissipated power in the photodiode, the optical power can be calculated from three slightly different bridge's voltage signals by using the equation:

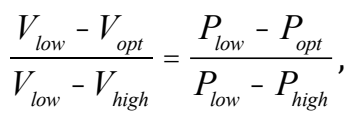

where $V_{\text {low }}, V_{\text {high }}$ and $V_{\text {opt }}$ are three voltage signals corresponding to two known electrical power levels $\left(P_{\text {low }}\right.$ and $P_{\text {high }}$ respectively) and an unknown optical power, $P_{\text {opt }}$. Electrical powers can be calculated by measuring the forward bias voltage $V_{\text {bias }}$ and the current produced $I$ :

$$
P_{\text {high,low }}=I_{\text {high,low }} V_{\text {bias }_{\text {high,low }}},
$$

The self-calibration routine in thermal mode consists of the following steps:

- In absence of radiation, forward bias the photodiode producing an electrical heating slightly below the optical heating and measure $V_{\text {low }}$ and calculate $P_{\text {low }}$.

- Then switch off the forward bias and irradiate the photodiode with optical power keeping it in open circuit and measure $V_{\text {opt }}$.

- Then shut down irradiation and forward bias the photodiode to produce an electrical heating slightly higher than the optical one and measure $V_{\text {high }}$ and calculate $P_{\text {high }}$.

- Then repeat step 2 (optical power) to have a more symmetrical situation of heating and cooling cycles.

Bearing in mind that the time constant of the system is around 1 minute, the power (either optical or electrical) should be applied for at least five times the time constant in order to achieve a stable saturation level on the bridge's voltage signal. Moreover, the sequence is repeated for 50 cycles in order to select a steady range of the signal along the time. Fig. 5 shows an example of the bridge's voltage in every step.

Fig. 5 shows the decreasing trend of the bridge's output due to the cumulative heating of the system along the heating cycles. The drift can be removed by fitting it to a low order polynomial and subtracting the base line from the data. However, there are drifts, caused by other unknown effects within each cycle. 


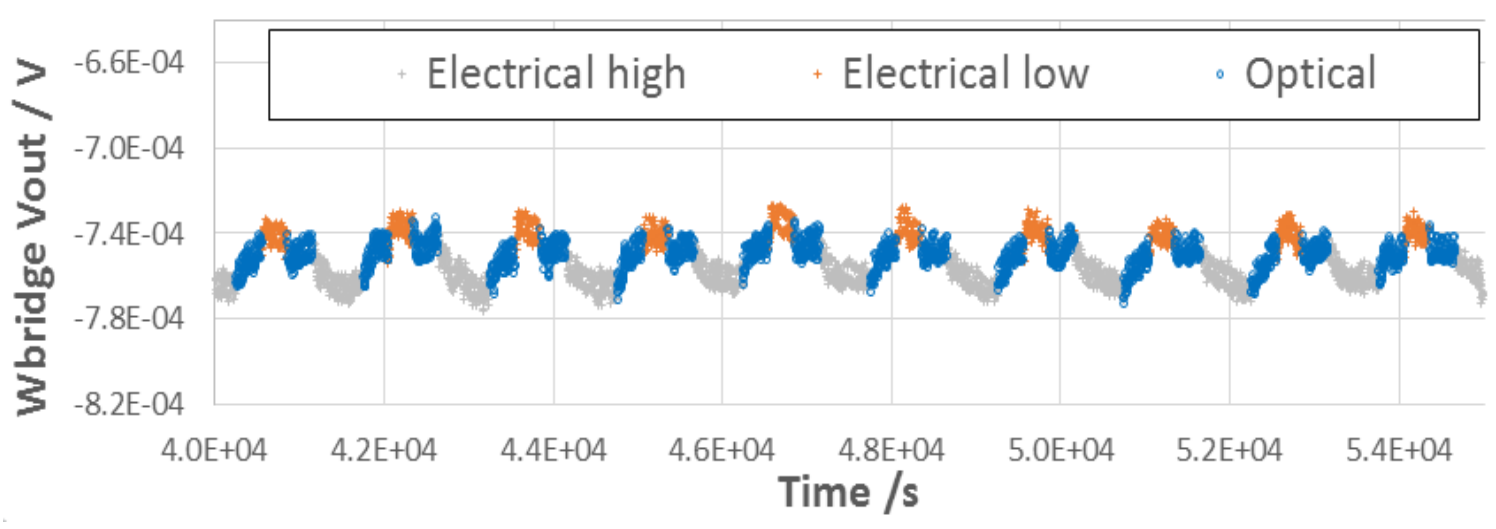

Fig. 5. Bridge voltage along the measurement sequence of electrical and optical power in thermal mode around $1 \mathrm{~mW}$.

The voltage signal of the highest electrical power ( $V_{\text {high, }}$ in grey) has the largest drift, that is the reason why this is the signal measured for a longer time, ten times the time constant, waiting for a steady output. On the other hand, the voltage signal of the lowest electrical power ( $V_{\text {low }}$ in red) seems quite steady. The optical signal ( $V_{\text {opt }}$, in blue) was measured in between in order to avoid big steps of cooling or heating in each cycle.

There is another characteristic trend worth noticing: optical heating signal going from high electrical to optical is drifting much more than going from low electrical to optical. Low electrical is relatively stable. High electrical heating signal has the most pronounced drift close to the optical signal going from high electrical. One explanation of these characteristics may be that the power difference between the optical and lower electrical are closer than the optical and the higher electrical. This may indicate that the procedure could be improved as the contribution to the standard deviation is most likely drift and not random noise.

The measured voltage signals, $V_{\text {low }}, V_{\text {high }}$ and $V_{\text {opt }}$ were processed, smoothing the signals by filtering some noisy scattered data points and calculating a moving average in order to estimate an average value of each signal. After smoothing the signals, the standard uncertainty of the average value of the measured signals is lower than $1 \%$ for power levels higher than $300 \mu \mathrm{W}$.

At the end of the thermal mode routine, the short-circuit photocurrent produced under the same optical irradiation, $I_{p h}$, is measured.

\section{Results and discussion}

Results shown in Table 1 below correspond to irradiating the device with a beam from an argon laser at the wavelength of $488 \mathrm{~nm}$, following the routine described in section 3 for different optical power levels.

TABLE 1. Values of calculated optical power and their corresponding measured photocurrent.

\begin{tabular}{|c|c|}
\hline \hline Calculated optical power & Measured photocurrent \\
\hline \hline $305.17 \mu \mathrm{W}$ & $108.31 \mu \mathrm{A}$ \\
$552.21 \mu \mathrm{W}$ & $181.23 \mu \mathrm{A}$ \\
$717.29 \mu \mathrm{W}$ & $233.04 \mu \mathrm{A}$ \\
$1054.52 \mu \mathrm{W}$ & $332.73 \mu \mathrm{A}$ \\
$1502.69 \mu \mathrm{W}$ & $459.09 \mu \mathrm{A}$ \\
$4102.73 \mu \mathrm{W}$ & $1198.43 \mu \mathrm{A}$ \\
\hline \hline
\end{tabular}

Hence, the responsivity of the device can be estimated by representing the measured photocurrent versus the calculated optical power in thermal mode and fitting the points to a linear function.

The linear fit to all the points gives a very high regression coefficient (see Table 2), which indicates that the highest power level could be outside the linear response range of the photodiode. To check this, linear regression was calculated for subsets three and four points as well, excluding the highest power levels. Regression coefficients for these data subsets are also shown in Table 2. Although the difference on the regression coefficient is not relevant, the first four points subset was chosen to estimate the responsivity. 
TABLE 2. Comparison of the coefficients of three linear regressions.

\begin{tabular}{|c|c|}
\hline \hline Linear regression & $\mathrm{R}^{2}$ \\
\hline \hline 3 first points & 0.999713798 \\
4 first points & 0.999898834 \\
5 first points & 0.999702121 \\
\hline
\end{tabular}

Fitting the first four points, the equation of the linear regression, as it is shown in the Fig. 6, provides the value of the slope which is the responsivity of the photodiode according to equation (1). The linear regression shows a y-intercept, although this value should be equal to zero according to equation (1). This might be due to a remaining offset unbalanced in the Wheatstone bridge which would produce a nonequivalence between the two modes.

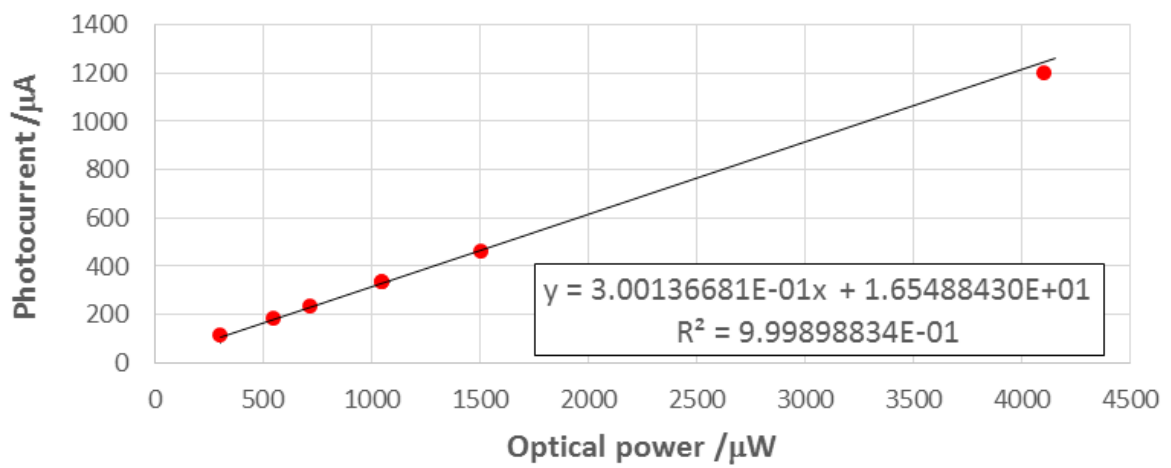

Fig. 6. Photocurrent measured versus optical power calculated and linear regression of the first four points representing the linear domain of the response of the device.

The ratio between the measured photocurrent and the photocurrent estimated from the linear fit at the same power levels is given in Table 3 and plotted versus the measured photocurrent in Fig. 7. The first four points in this figure, which correspond to optical power values from $300 \mu \mathrm{W}$ to $1 \mathrm{~mW}$, are scattered around 1 , so they could be considered in the linear domain, since their error bars include the value 1 , but the other 2 (not used for the linear fitting) would appear to be outside the linear range of the photodiode.

TABLE 3. Ratio between the measured photocurrent and the photocurrent given by the linear regression.

\begin{tabular}{|c|c|}
\hline \hline Photocurrent linear fitting & Relative difference between photocurrents \\
\hline \hline $108.14 \mu \mathrm{A}$ & 1.00154135 \\
$182.29 \mu \mathrm{A}$ & 0.99419370 \\
$231.83 \mu \mathrm{A}$ & 1.00520258 \\
$333.05 \mu \mathrm{A}$ & 0.99905598 \\
$467.56 \mu \mathrm{A}$ & 0.98188815 \\
$1247.93 \mu \mathrm{A}$ & 0.96033665 \\
\hline
\end{tabular}

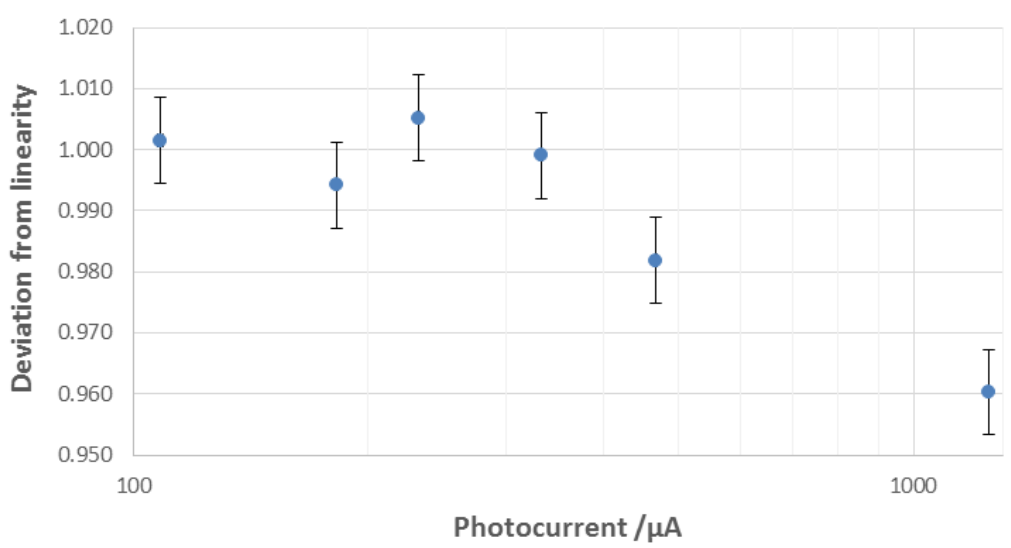

Fig. 7. Deviation from the linear behaviour of the device versus the photocurrent. 
At this point, calculating the IQE of the photodiode from equation (1) can be used as estimator of the accuracy in the method and try to highlight whether the short circuit photocurrent is non-linear or the equivalence between the optical and electrical heatings is overestimated. To achieve this, it is essential to know the responsivity and the reflectance of the optical elements.

For the case of the present configuration of the device, the fraction of radiation non-absorbed in the photodiode can be estimated as it is described in equation (4):

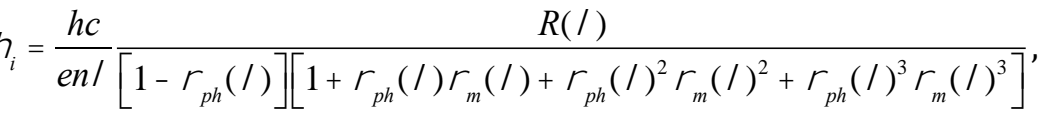

where $\rho_{m}$ is the reflectance of the mirror and $\rho_{p h}$ the reflectance of the photodiode and both depend on the wavelength of the incident radiation.

The trap configuration of the mirror, placed in an angle of $15^{\circ}$ relative to the photodiode, allows for collecting in the photodiode three reflections from the mirror as well as the incident beam (see the optical path sketched in Fig. 1) with an incidence angle of $45^{\circ}$ and assuming the device perpendicularly aligned to the beam. Considering a typical reflectance value of an aluminium mirror [10] and a typical reflectance value of similar photodiodes $(0.335$ at $488 \mathrm{~nm})$ the non-absorbed radiation can be estimated.

If the slope obtained in the linear regression in Fig. 6 is employed as responsivity value $(0.3001 \pm 0.0021)$ $\mathrm{A} / \mathrm{W}$ at $488 \mathrm{~nm}$, the IQE calculated for the photodiode is $0.825 \pm 0.005$, which is a low value. Then the thermal equivalence would seem to be overestimated, or the losses of photo-generated charge carrier are large in the n-doped emitter layer during photocurrent measurement.

\section{Conclusions}

The device built is able to work in the linear domain, in thermal and photocurrent modes within a range of optical power between $300 \mu \mathrm{W}$ and $1 \mathrm{~mW}$. Lower power values are limited by the signal-to-noise ratio, and higher values are limited by the nonlinear behaviour between the two modes of the system. However, this power domain could be extended by applying a sufficiently large reverse bias during photocurrent measurements in a future improvement.

The evaluation of the results shows the feasibility to experimentally predict the IQE of a silicon $n p$ photodiode at room temperature, using temperature sensors with an uncertainty under $1 \%$, although some refinement is needed.

\section{Acknowledgements}

This work has been supported by the European Commission through the project SIB57-NEWSTAR under the European Metrology Research Programme (EMRP). The EMRP is jointly funded by the EMRP participating countries within EURAMET and the European Union. The authors of CSIC acknowledge support from the Comunidad de Madrid and the European Union under program SINFOTON-CM (S2013/MIT-2790).

Parts of this work have been presented at XI Reunión Nacional de Óptica in Self-calibration of silicon photodiodes internal quantum efficiency by using temperature sensors, 2015. 